\title{
Community Violence Exposure, Social Cognition, and Aggression Among Urban Elementary School Children
}

\author{
Nancy G. Guerra, L. Rowell Huesmann, and Anja Spindler
}

\begin{abstract}
The effects of witnessing community violence on aggressive cognitions and behavior were investigated in an ethnically diverse sample of 4,458 children living in urban neighborhoods. Prior violence exposure had a significant effect in increasing aggression, normative beliefs about aggression, and aggressive fantasy. Although exposure to violence predicted aggressive behavior both in Grades 1 through 3 (ages 5-8) and Grades 4 through 6 (ages 9-12), the effects on social cognition were only evident in the later grades. Furthermore, the effect of violence exposure on aggression in the later grades was partially mediated by its effect on social cognition. These findings suggest that witnessing community violence has an effect on children's aggressive behavior through both imitation of violence and the development of associated cognitions as children get older.
\end{abstract}

One of the most robust findings in the literature on the etiology of aggression is the statistical continuity of aggression from early childhood into adulthood (Huesmann, Eron, Lefkowitz, \& Walder, 1984; Olweus, 1979). Several researchers have argued that one of the mediating factors in maintaining this continuity is the pattern of social cognition the child develops supporting aggression. In other words, aggressive behavior is seen as being increasingly controlled by internal self-regulating processes that become more stable over time (Huesmann \& Guerra, 1997). Huesmann $(1988,1998)$ has emphasized the importance of cognitive schemas used as models of the world, cognitive scripts stored in memory and used as guides for social behavior, and normative beliefs used to evaluate the appropriateness of scripts. The more aggressive child is presumed to have acquired cognitive schemas depicting the world as a more hostile place, to endorse normative beliefs that aggression is more acceptable, and to have encoded in memory more extensive, wellconnected networks of social scripts emphasizing aggressive responses.

Nancy G. Guerra, Department of Psychology, University of California, Riverside; L. Rowell Huesmann and Anja Spindler, Institute for Social Research, University of Michigan.

Funding for this work has been provided by a grant from the National Institute of Mental Health to the first author and by the Centers for Disease Control to the second author. The authors wish to thank Matheos Yosef and Steve Raudenbush for advice and assistance in the data analyses and to thank Leonard Eron, David Henry, Patrick Tolan, RichardVan Acker, and the large staff of the Metropolitan Area Child Study for assistance in collecting the data.

Correspondence concerning this article should be addressed to Nancy Guerra, Department of Psychology, University of California, Riverside, CA 92521. Electronic mail may be sent to Nancy.Guerra@ucr.edu.
These cognitions are learned over time as the child navigates a variety of social contexts. From a social learning perspective, an important component of this learning process is the extent to which a child observes the aggressive behavior of powerful role models (Bandura, 1973; Eron, 1987). Social-cognitive reformulations and other theories emphasizing social information processing further highlight the mediating role of cognition in understanding the link between observation of aggression and aggressive behavior (Bandura, 1986; Dodge, Bates, \& Pettit, 1990). Children observe what goes on around them; make inferences and attributions; and acquire scripts, schemas, and normative beliefs that serve as guides for future behavior.

Of course, learned cognitions interact with emotional processes to influence aggressive behavior. Aversive stimulation in the form of provocations and frustrations is an important situational precursor of aggression, and contexts for growing up vary widely in such stimulation. Huesmann (1998) has argued that children who are repeatedly exposed to violence during childhood habituate to it and experience it as less aversive. This makes it easier for them to think about and plan aggression. Additionally, individual differences in emotional responsivity and emotion regulation may predispose some children to be more at risk than others. For instance, for children who are high in emotionality, arousing events such as violence exposure may make it more difficult for them to develop the regulatory mechanisms necessary to control their emotions (Eisenberg, Fabes, Nyman, Bernzweig, \& Pinuelas, 1994). Without such emotional control, their risk for aggressive and

(C) 2003 by the Society for Research in Child Development, Inc. All rights reserved. 0009-3920/2003/7405-0020 
antisocial behavior increases. Because of both these cognitive and emotional processes associated with exposure to violence, children who have had more opportunities to observe violence across a range of contexts should be at greater risk to develop aggressive habits of their own.

Over the last decade, several studies have attempted to document the extent of children's exposure to community violence. These studies consistently have pointed to alarmingly high rates, particularly in high-crime inner-city and urban neighborhoods. For example, in a study of African American youth in Chicago, approximately 65\% of school-age children reported having witnessed a serious assault and 33\% had witnessed a homicide (Bell \& Jenkins, 1991). In another study of youth living in New Haven, Connecticut, $40 \%$ of the children surveyed reported witnessing one or more violent crimes in the past year (Marans \& Cohen, 1993). Other studies using mother's reports of children's exposure have pointed to even higher rates of witnessing at least some type of violence in inner-city communities (Osofsky, Wewers, Hann, \& Fick, 1993; Richters \& Martinez, 1993). Such findings have resulted in comparisons of these high-violent communities with war zones and have resulted in repeated calls for an examination of the effects of exposure to chronic violence and danger on children's development (Garbarino, Kostelny, \& Dubrow, 1991; Lynch \& Cicchetti, 1998).

Indeed, there is also a growing body of research linking exposure to community violence with subsequent disruptions in children's well-being. Several studies have found a relation between violence exposure and mental health problems. For example, in one study, more than $50 \%$ of children exposed to violence before age 10 developed later psychiatric problems (Martinez \& Richters, 1993). Mental health problems associated with violence exposure typically include increases in depression, anxiety, dissociation, and other trauma-related symptoms (Singer, Anglin, Song, \& Lunghofer, 1995) as well as problems in emotion regulation (Pynoos, 1993). Of particular relevance to the current investigation are studies that examine the effects of community violence exposure on aggressive and antisocial behavior. Several studies have found contemporaneous and longitudinal relations between witnessing community violence and aggressive and antisocial behavior in children and adolescents. For example, Singer et al. (1999) found that exposure to community violence predicted violent behavior among urban African American and White youth in Grades 3 through 8. Similarly, Schwab-Stone et al. (1999) reported a strong relation between violence exposure and externalizing symptoms 2 years later for children in Grades 6, 8, and 10. Other studies also have reported higher levels of violence exposure among dangerously violent adolescents (Flannery, Singer, \& Wester, 2001).

Although these studies point to a relation between community violence exposure and emotional and behavior problems including aggression, four issues are worth mentioning. First, violence exposure typically has been defined broadly, including both observation of violence (i.e., witnessing violence) and direct experience (i.e., violent victimization). Yet, the correlates, consequences, and mechanisms of influence related to being a victim of violence may differ significantly from those related to witnessing violence. For example, in a recent study of fourth- through sixth-grade innercity children, Schwartz and Proctor (2000) found different effects for violence exposure and violent victimization. Specifically, synchronous correlations were found between witnessing community violence and positive outcome and efficacy beliefs for aggression as well as the perception that aggression is an appropriate response to ambiguous peer provocation. These relations were not obtained when examining the effect of exposure to violence through direct victimization, which was linked instead to problems in emotion regulation and related social difficulties.

Second, many of the studies linking violence exposure and aggressive and violent behavior either have focused on violence at home or have not distinguished it from violence in the neighborhood. Similarly, studies looking at the effect of violence exposure on social-cognitive processes typically have looked at the relation between family experiences such as harsh discipline on patterns of social information processing (Weiss, Dodge, Bates, \& Pettit, 1992). Although there has been considerable empirical support for the relation between abuse and other forms of domestic violence on subsequent aggressive, delinquent, and violent behavior (Widom, 1989; Rivera \& Widom, 1990), as well as the effects of abuse on children's social information processing (Dodge et al., 1990; Spacarelli, Coatsworth, \& Bowden, 1995), caution should be exercised in extending these findings to the effects of community violence exposure.

Third, studies of the prevalence and effects of community violence exposure on children generally have focused either on a specific age group or on a broad range of ages and have not examined differences as a function of the child's developmental level. 
Indeed, the majority of studies of community violence exposure and aggression have been limited to middle school and high school youth, with studies of younger children more commonly examining the combined impact of living in violent communities and violent homes (Osofsky, 1995). Yet, our theoretical model linking aggressive behavior with aggressive scripts and normative beliefs that are learned, in part, via exposure to aggressive models, suggests that the influence of contextual factors such as living in a violent setting should be most pronounced during the elementary school years when these characteristic patterns of cognition are developing.

In previous study examining the reciprocal relations between normative beliefs about aggression and aggressive behavior during the early and late elementary school years, Huesmann and Guerra (1997) found that aggressive behavior predicted aggressive beliefs during the early elementary school years, and aggressive beliefs predicted behavior during the later elementary school years. In other words, children's cognitions about the legitimacy of aggression emerged from observations of their own aggressive behavior in the early grades, with these beliefs serving as guides for behavior during the later elementary grades. Given that social cognitions become more stable during the later elementary years, it may be that observation of events (including one's own social interactions as well as other events across different contexts) relates to the development of social-cognitive structures during early childhood but that these social-cognitive structures, once formed, are less sensitive to new information from the environment.

Fourth, although studies have documented a relation between violence exposure and aggression, very little attention has focused on simultaneously examining mechanisms that may help explain this relation. Clearly, social-cognitive influences on aggression have been studied extensively. However, little research has examined specifically the extent to which community violence exposure affects aggressive behavior via its impact on social cognition. Several researchers have suggested this causal chain; for instance, Ng-Mak, Salzinger, Feldman, and Stueve (2002) posit a normalization of violence theoretical model wherein normalizing cognitions about violence serve as mediators between violence exposure and its behavioral consequences.

In the current study, we examined the development and continuity of community violence exposure, aggressive cognitions, and aggressive behavior as well as the concurrent and longitudinal relations among these variables in a multiethnic sample of elementary school children living in high-violence, urban communities. In particular, we were interested in determining the role of exposure to community violence on children's aggressive cognitions and in examining the mediating role of social cognitions in explaining the hypothesized link between violence exposure and aggression. We were also interested in examining whether these relations were different across age and gender.

We focused on two components of social cognition that have been theoretically linked to the development of aggressive behavior and that should be influenced by observation of violent events: fantasizing about aggressive scripts and normative beliefs about aggression. These cognitions normalize violence and, hence, help children adapt to the stress and trauma of witnessing violent events. However, they should also make it more likely that children will subsequently behave more aggressively (Huesmann, 1998; Huesmann \& Guerra, 1997). For instance, children who engage in aggressive fantasies that involve cognitive rehearsal of aggressive actions will be more likely to retrieve these scripts subsequently. Believing that aggression is more normative will make it more likely that they will use a retrieved aggressive script. Similarly, viewing violence as normative may desensitize children to its true consequences, resulting in a streetwise mentality that has been described by some as "pathologic adaptation" (e.g., Schwab-Stone et al., 1995).

Based on our theoretical model and the rationale presented previously, we addressed five issues:

1. Does exposure to community violence, aggressive cognitions, and aggressive behavior increase or decrease on average during the elementary school years? In the context in which these urban children are growing up, we expected most children's aggressive cognitions and aggression to increase on average. However, exposure to community violence involves the interplay of actual violence in the community with opportunities to observe this violence that may be related to practical considerations (e.g., whether parents allow children to play outside); therefore, its trajectory is harder to predict.

2. Is there significant continuity in exposure to violence, aggressive cognitions, and aggressive behavior during the elementary school years, and if so, is this continuity more evident during the later elementary years? We expected to find substantial continuity of aggressive behavior and 
aggressive cognitions in childhood, particularly in the later years as prior studies have shown. However, whether there is a continuity of exposure to violence in these children is harder to predict.

3. Does exposure to community violence predict subsequent aggressive behavior and subsequent aggressive cognitions? Do these relations vary with age? Is there any evidence that aggressive cognitions or aggressive behavior also stimulate significantly more exposure to community violence? We expected that children who view violent exchanges more regularly in the community should subsequently be more likely to fantasize about them, view them as normative and acceptable, and mimic them in their behaviors. In terms of age differences, previous studies of observation of one's own aggressive behavior suggest that the effects of violence exposure on cognition should be stronger during the early years while patterns of thought are developing (e.g., Huesmann \& Guerra, 1997). However, it may be that observation of community violence and related cognitive shifts occur later in childhood when children are developing adaptive coping strategies (e.g., Dempsey, 2002). Thus, it is hard to predict specific age differences. Finally, we expected that aggressive behaviors and cognitions might have a reciprocal influence on increasing a child's risk for exposure to community violence. More aggressive children who are more accepting of violence should be more willing to place themselves in situations where they might observe violence.

4. Do social cognitions mediate the relation between community violence exposure and subsequent aggression? Are these mediational influences stronger for older children than for younger children? We predicted that exposure to community violence would result in cognitions that normalize aggression, which would then prompt children to behave more aggressively. In terms of age differences, we expected that mediation would be stronger for older children, given the greater stability of cognitions during the later years and their increased role in stimulating subsequent behavior.

5. Does exposure to community violence have differential effects on males and females? Studies that have looked at relations between exposure to community violence and aggression among elementary school children have not found any consistent pattern in gender differences (Attar,
Guerra, \& Tolan, 1994; Schwartz \& Proctor, 2000). Although boys generally are more aggressive than girls, particularly in relation to physical aggression, and are more likely to view aggression as normative and acceptable, this does not mean that the impact of witnessing violence in terms of modeling or associated cognitions will necessarily be different between boys and girls. Thus, we did not expect to find gender differences in any of the hypothesized relations.

\section{Method}

The data for the analyses came from the Metropolitan Area Child Study, a 6-year longitudinal study of multiple cohorts of children as they moved from the first to sixth grades in 21 Chicago area schools (Metropolitan Area Child Research Group, 2002). About $75 \%$ of the children in the study received an intervention intended to reduce their aggression at one or two times during the 6 years. However, independently of the intervention, data were collected on many other measures repeatedly at 1-year intervals during the study, yielding from one to six waves of longitudinal data on each child. It is these data that were used to address the major aims of this study.

\section{Participants}

The Metropolitan Area Child Study sample, which was used in our analyses, consists of 4,458 children who were in the first to sixth grades in 21 schools in the Chicago metropolitan area between 1991 and 1997. The sample falls into eight birth cohorts ranging from 1981 to 1988. The sampling unit was the school and the project selected schools that volunteered from neighborhoods with high levels of social distress and economic disadvantage in the Chicago metropolitan area. For a detailed description of school recruitment procedures, see Guerra, Huesmann, Tolan, Van Acker, and Eron (1995).

Once the schools were selected, parental permission was solicited in the initial year of the study for every child in the first through fourth grades and in subsequent years for every child entering the first grade in the selected schools. By the end of the data collection in 1997, parental permission for participation had been received for 4,471 children, about $85 \%$ of the eligible children. About $40 \%$ of the sample was African American, 40\% was Hispanic, and about 20\% was non-Hispanic White. The sample consisted of 
2,247 males and 2,211 females, with 13 participants of unidentified gender being dropped, resulting in a total of 4,458 participants. The sample was predominately lower socioeconomic status with more than two thirds of the children qualifying for free lunch programs. The unemployment rate in the selected Chicago neighborhoods was $29.4 \%$ according to the 1990 census, and in the Aurora neighborhoods it was $19.7 \%$. The respective per capita crime rates were 219 per 100,000 in the Chicago neighborhoods and 113 per 100,000 in the Aurora neighborhoods.

Attrition over 6 years of the study occurred at both the school and individual levels. A total of 16 schools started the study. Of these schools, 5 dropped out at some point because of changes in administrative interest or teacher support and 5 schools were added to replace them, resulting in our total of 21 schools. However, data collected from a school before that school withdrew from the study could be included in many of our growth curve analyses as described later. Individual participant attrition within schools also occurred because of the high mobility of the population. Because the school was the sampling unit, students who left their original schools were not followed for future assessments. Additionally, during some years not all measures could be given to all participants because of school and teacher restrictions on time or interviewing difficulties. Together, these factors created a complicated pattern of available data for each cohort, which is illustrated in Table 1. It is difficult to summarize the attrition represented in this table with a single number, but, as an example, the average 3-year attrition in assessment of aggres- sion within a cohort from Grades 1 to 3 was about $27 \%$ and from Grades 4 to 6 was about 32\%.

These attrition figures are not unusual for studies in high-risk neighborhoods, but they place some limit on the generalizations that can be drawn from the data. A comparison of the retained participants and those who dropped out revealed that the dropouts had been slightly higher on initial aggressiveness, $S D=.19$ for third-grade dropouts, $t(1118)=4.14, p<.001$, and $S D=.11$ higher for sixth-grade dropouts, $t(1378)=2.18, p<.05$. This finding is also typical for longitudinal studies of aggression. A possible consequence of such attrition is that the magnitude of relations with aggression may be underestimated because of a restriction in range of the aggression scores at the high end.

The amount of missing data created by the attrition and the multiple cohort design presents problems for many analyses techniques. However, by using recent growth-curve-modeling techniques that are robust for missing data, we can surmount these difficulties and estimate effects over all six waves. For subsets of grades (e.g., Grades 1-3 or 4-6), the pattern of missing data presents less of a problem, and structural modeling can also be used.

\section{Measures}

Although a large number of child measures were obtained during this project, only three measured constructs are relevant for the current study: children's aggressive behavior, children's exposure to neighborhood violence, and children's social cognitions. The composition of these measures is summarized in Table 2.

Table 1

Design of the Longitudinal Study: Sample Sizes by Cohort and Grade for 4,458 Children in 21 Chicago Area Schools

\begin{tabular}{|c|c|c|c|c|c|c|c|c|c|c|c|c|c|c|c|c|c|c|}
\hline \multirow[b]{3}{*}{ Birth cohort } & \multicolumn{18}{|c|}{ Sample sizes for measures AG, NV, and SC in each grade } \\
\hline & \multicolumn{3}{|c|}{ 1st grade } & \multicolumn{3}{|c|}{ 2nd grade } & \multicolumn{3}{|c|}{ 3rd grade } & \multicolumn{3}{|c|}{ 4th grade } & \multicolumn{3}{|c|}{5 th grade } & \multicolumn{3}{|c|}{ 6th grade } \\
\hline & AG & NV & SC & AG & NV & SC & AG & NV & SC & AG & NV & SC & AG & NV & SC & AG & NV & SC \\
\hline 1988 & 412 & & 400 & & 393 & 394 & 346 & 278 & 323 & & & & & & & & & \\
\hline 1987 & 311 & & 281 & & 275 & 270 & 224 & 216 & 211 & & & & & & & & & \\
\hline 1986 & 337 & & 283 & & 301 & 297 & 267 & 240 & 223 & & & & & & & & & \\
\hline 1985 & 590 & & & 101 & 523 & 528 & 462 & 409 & 400 & & & & & 176 & 177 & 203 & 191 & 184 \\
\hline 1984 & 706 & 225 & 612 & 478 & 266 & 500 & 398 & 407 & 382 & & & & & 220 & 221 & 232 & 216 & 211 \\
\hline 1983 & & & & 668 & 218 & 541 & 478 & 300 & 500 & 407 & 408 & 380 & & 247 & 246 & 253 & 240 & 239 \\
\hline 1982 & & & & & & & 521 & & & 638 & 499 & 525 & 105 & 545 & 546 & 497 & 453 & 457 \\
\hline 1981 & & & & & & & & & & 712 & 208 & 602 & 509 & 325 & 543 & 446 & 448 & 419 \\
\hline Total & 2,356 & 225 & 1,576 & 1,247 & 1,976 & 2,530 & 2,696 & 1,850 & 2,039 & 1,757 & 1,115 & 1,507 & 614 & 1,513 & 1,733 & 1,631 & 1,548 & 1,510 \\
\hline
\end{tabular}


Table 2

Measures

Teacher's report aggression

(Achenbach, 1978)

Peer-nominated aggression

(Eron, Walder, \& Lefkowitz, 1972)

Composite aggression

Exposure to neighborhood violence (Attar, Guerra, \& Tolan, 1994)

\footnotetext{
Aggressive fantasy

(Rosenfeld, Huesmann Eron, \&

Torney-Purta, 1982)

Normative beliefs Approving

Aggression

(Huesmann \& Guerra, 1997)
}

Sum of 25 items on the aggression scale of Achenbach's Child Behavior Checklist $(\mathrm{CBC}$; range $=0-50)$.

Proportion of times nominated by classroom peers on 10 questions of the form:

Who starts a fight over nothing?

Who tells stories and lies to get other children in trouble?

Who pushes or shoves children?

Who says mean things?

Average of standardized scores of teacher's CBC aggression and peer-nominated aggression

Average of four self-report items (yes $=1$; no $=0$ ) of the form "During the last year..."

1. Have you seen anyone beaten shot or really hurt by someone?

2. Have you seen or been around people shooting guns?

3. Have you been afraid to go outside and play, or have your parents make you stay inside because of gangs or drugs in your neighborhood?

4. Have you had to hide someplace because of shootings in your neighborhood?

Average of 6 self-report items (no $=1$; a lot $=3$ ) of the form:

Do you sometimes have daydreams about hitting or hurting someone you don't like?

Do you play games where you pretend to fight with someone?

Average of 20 self-report items (perfectly $\mathrm{OK}=4$; really wrong $=1$ ).

12 are of the form:

Suppose a boy says something bad to another boy John - do you think it's OK for John to hit him?

8 are of the form:

In general, it is wrong to hit other people.
Children's aggressive behavior was assessed from two sources: peers and teachers. Classmates' nominations were used to assess aggression through the Peer Nomination Inventory (Eron, Walder, \& Lefkowitz, 1972). Children were asked to nominate their classmates who engage in 10 different physical, verbal, and indirect aggressive behaviors. The scores represent the ratio of times a child is nominated out of times the child could have been nominated. The scale's reliability (.95 to .97 ) and validity have been demonstrated in numerous studies (e.g., Guerra et al., 1995; Huesmann et al., 1984). For classrooms in which peer nominations could not be collected (23.2\% of all assessments), we used the Teachers Predictions of Peer-Nominations Scale, which has been shown to provide unbiased, highly reliable (coefficient alpha $=.97$ ), and valid estimates of the actual peer nominations (Huesmann, Guerra, Eron, \& Crawshaw, 1994). For the second assessment of aggressive behavior for all children we used the Teacher Report Form (TRF) of the Child Behavior Checklist (Achenbach, 1978, 1991). The aggression scale of the form consists of 25 behaviors that the teacher rates on a 3-point scale. The scale and measure are well validated and reliable. Coefficient alpha for the TRF aggression scale is .96 .

We combined these two scales to form a composite aggression score by first standardizing each measure across all scores across all years and then taking the average of the standardized scores. Thus, the standardized aggression score represents a deviation score from the grand mean aggression score for all observations on all children in all grades. This centering of the scale allows for more meaningful growth curve analysis without changing any of the correlations between variables. In the intervention analyses reported earlier (Metropolitan Area Child Study Research Group, 2002), we used a different composite aggression score that corrected for the two differing distributions of component scores to form a more reliable composite. However, that score could not be computed if either component score was missing. The current composite can be computed from just one component, resulting in a higher sample size for analysis.

Two social cognition measures were obtained through children's interviews. Aggressive fantasies were assessed with the aggressive fantasies subscale of the Children's Fantasy Inventory (Rosenfeld, Huesmann, Eron, \& Torney-Purta, 1982). This is a self-report measure developed for assessing the nature and frequency of fantasy production in school-aged children. It was designed to measure cognitive rehearsal of different action sequences or scripts (e.g., prosocial, aggressive). The aggressive 
subscale in particular is intended to assess how often children rehearse aggressive scripts, making them more readily accessible and likely to be used in social problem solving (Huesmann, 1988, 1998). The aggressive fantasy scale consists of six items such as "Do you sometimes have daydreams about hitting or hurting somebody that you don't like?" Children responded to these items on a 3-point scale (no, a little, or a lot), and responses were averaged over the six items. Cronbach's alpha for this measure was .66.

Normative beliefs about aggression were measured using the Normative Beliefs about Aggression Scale (Huesmann \& Guerra, 1997; Huesmann, Guerra, Miller, \& Zelli, 1992). This is a reliable and wellvalidated self-report measure that assesses children's perception of how acceptable it is to behave aggressively both under varying conditions of provocation and when no conditions are specified. Children endorse 20 items (e.g., "If you're angry, it is OK to say mean things to other people") by means of a 4point scale (e.g., perfectly OK, sort of OK, sort of wrong, or really wrong). The total score is the average of all items. Cronbach's alpha for this scale is .87 .

Children's exposure to neighborhood violence was measured with a subscale of the stressful events scale developed by Attar et al. (1994). This is a fouror five-item scale (depending on the year) assessing the extent to which children have been exposed to violence in the community setting. The questions ask the children whether "during the last year" they have had to hide someplace because of shootings in the neighborhood, have had to stay inside because of gangs or drugs in the neighborhood; have seen people shooting guns; have seen anyone beaten, shot, or really hurt by someone; or have had someone they know been beaten, attacked, or really hurt by others. Each question is scored either 0 (no) or 1 (yes), and a child's exposure to neighborhood violence score is the average of these scores. The internal consistency of the subscale is .61. Other studies of children's violence exposure have used this type of brief, yes - no format and have reported similar internal consistencies (e.g., Schwab-Stone et al., 1995). In previous research, Attar et al. (1994) found that children's scores on this scale were related to actual levels of serious community violence as measured by official crime data, indicating that the scale is also a valid measure of community violence exposure.

\section{Procedure}

Each cohort of children was assessed on the three constructs according to the schedule shown in Table
1. The intention was to assess each child every spring from the first through sixth grades, but the oldest cohorts were only assessed from the fourth to sixth grades and the youngest cohorts from the first through third grades.

Most assessments occurred in the spring of the year when the children were completing the designated grade; however, a few occurred in the subsequent fall when the children were actually just starting the next grade. Nevertheless, these are clustered with the previous grade's data. Thus, the data-collection periods span up to 6 months and are separated from the next data-collection period by at least 6 months and not more than 18 months. All assessments of the children were done in school and were done as individual oral self-report assessments for children in the first grade but as classroom paper-and-pencil assessments for children in the older grades. All interviewers were trained graduate students, and for the classroom assessments, at least three interviewers were present with two roaming the classroom to help individual children with their answers. If children were absent on the assessment day, a make-up individual or small-group assessment was scheduled. For bilingual or monolingual Spanish-speaking classrooms, all measures were translated into Spanish using back-translation methods. Spanish-speaking interviewers administered the measures to these classrooms and children. No difficulties in understanding the assessments were noted.

\section{Results}

\section{Growth in Aggression, Cognitions Supporting Aggression, and Exposure to Violence}

The mean scores for each of the variables across grades are shown in Table 3. However, these scores can be misleading for estimating growth because of the changing sample composition and missing data as illustrated in Table 1. In addition, the clustering of participants within schools introduces dependencies that violate the usual assumption of independence of observations. The accepted modern technique for dealing with such data is to estimate developmental growth across years using a hierarchical linear growth curve model (HLM) with time as the first level, child as the second level, and school as the third level (Bryk \& Raudenbush, 1987; Raudenbush \& Chan, 1992; Rogosa, Brandt, \& Zimowski, 1982). These initial models included only grade as a predictor variable with random error contributed by school and child within school.

The resulting calculated growth curve for exposure to neighborhood violence showed that self- 
Table 3

Mean Scores by Grade

\begin{tabular}{|c|c|c|c|c|c|c|c|c|c|c|c|c|}
\hline \multirow[b]{3}{*}{ Grade } & \multicolumn{3}{|c|}{ Composite aggression } & \multicolumn{3}{|c|}{ Exposure to violence } & \multicolumn{3}{|c|}{ Aggressive fantasy } & \multicolumn{3}{|c|}{ Approving aggression } \\
\hline & \multicolumn{3}{|c|}{$($ range $=z)$} & \multicolumn{3}{|c|}{$($ range $=0-1)$} & \multicolumn{3}{|c|}{$($ range $=1-3)$} & \multicolumn{3}{|c|}{$($ range $=1-4)$} \\
\hline & $M$ & $N$ & $S D$ & $M$ & $N$ & $S D$ & $M$ & $N$ & $S D$ & $M$ & $N$ & $S D$ \\
\hline 1st grade & -.13 & 2,356 & 0.79 & .43 & 230 & .27 & 1.69 & 2,104 & .46 & 1.58 & 1,576 & .50 \\
\hline 2nd grade & .03 & 1,386 & 0.98 & .33 & 1,976 & .26 & 1.70 & 2,533 & .50 & 1.67 & 2,530 & .57 \\
\hline 3rd grade & .04 & 2,696 & 0.94 & .31 & 1,850 & .25 & 1.78 & 2,077 & .50 & 1.69 & 2,079 & .58 \\
\hline 4th grade & .05 & 1,857 & 0.99 & .30 & 1,197 & .26 & 1.98 & 1,509 & .47 & 1.91 & 1,509 & .56 \\
\hline 5 th grade & .16 & 703 & 1.13 & .27 & 1,514 & .24 & 1.93 & 1,736 & .46 & 1.91 & 1,734 & .57 \\
\hline 6th grade & .10 & 1,631 & 0.99 & .28 & 1,548 & .24 & 1.93 & 1,509 & .45 & 1.99 & 1,510 & .58 \\
\hline
\end{tabular}

reported exposure decreased significantly with grade, $B(30)=-.02$, HLM $t(20)=6.42, p<.001$, effect size $=0.10 S D$, with significant random variation also due to school, HLM $\chi^{2}(2746)=3029, p<.001$, and individual within school, HLM $\chi^{2}(20)=38.6, p<.01$. To test whether the grade effect was most likely due to an age effect or a time effect, we retested the model with birth cohort added as a predictor for each child. The slope of the growth curve for exposure by grade did not differ significantly by birth cohort, suggesting that the decrease in self-reported exposure cannot be explained by changing neighborhood environments during the study period. When gender was added to this growth model, we found that females reported more exposure to violence initially, $t(20)=2.63, p<.05$, but also experienced more of a reduction in exposure with age than did males, $t(20)=2.60, p<.05$. As we discuss later, this pattern of reduction in self-reported exposure may represent both a reduction in real exposure (e.g., females avoiding more violent contexts) and habituation to the perceptual salience of the violence.

Although self-reported exposure to neighborhood violence diminished as the child got older, comparable growth curve analyses showed that children's aggressive behavior and their social cognitions supporting aggression increased significantly with age. In standard deviation units, aggression increased about .04 SD per grade and the normative beliefs supporting aggression and aggressive fantasy both increased about .11 SD per grade. Males initially scored much higher than females on aggression, $t(20)=8.61, p<.001$, but their rate of growth was no different from females, $t(20)=.37$, ns. Males also initially scored significantly higher on normative beliefs supporting aggression, $t(20)=3.11, p<.01$, and on aggressive fantasizing, $t(20)=6.24, p<.001$, but on these social cognitions the patterns of growth did vary between genders. Females displayed a significantly greater increase in aggressive fantasizing with time, $t(20)=2.77, p<.01$, although their sample growth parameter for beliefs approving of aggression did not differ significantly from males. Again, we tested for cohort effects and found that the slope of the growth curves for aggression and the two social cognitions were not affected by cohort. However, the intercepts for the two social cognition variables decreased significantly for the more recent birth cohorts: normative beliefs, $t(20)=5.24, p<.001$, and aggressive fantasy, $t(20)=2.44, p<.05$. The judicious conclusion is that although there may have been trends during this period toward an overall reduction in social cognitions supporting aggression by successive cohorts, the average individual in all cohorts increased in aggression and social cognitions supporting aggression during the elementary school years.

Taken together, these growth curve results suggest that aggressive cognitions and aggressive behavior increase as a child grows older despite decreasing scores on exposure to neighborhood violence with age. These changes occurred in the early to mid 1990s, at the same time as overall scores on social cognitions supporting aggression and on exposure to neighborhood violence seemed to be decreasing slightly regardless of age.

\section{Continuity and Intercorrelations of Measures}

Within-variable longitudinal correlations were computed for 1- to 4-year lags between Grades 1 and 6. As is typical, aggressive behavior showed high continuity across these grade ranges, with 1-year correlations averaging .63, and with a .56 correlation for a 4-year lag. The other variables all displayed substantial year-to-year continuity ranging from .31 to .51, but the 4-year correlations, although significant, were not high, ranging from .14 to .17. In general, the year-to-year correlations for the social cognitions were higher in the later grades than in the earlier grades. 
Table 4

Intercorrelations of Aggression, Exposure to Violence, and Social Cognitions About Aggression in Second Grade (Above Diagonal) and Sixth Grade (Below Diagonal)

Correlations

Aggressive behavior Exposure to violence Aggressive fantasy Normative beliefs approving aggression

\begin{tabular}{|c|c|c|c|c|}
\hline \multicolumn{2}{|l|}{ Aggressive behavior } & $.18^{* * *}$ & $.14^{* * *}$ & $.16^{* * *}$ \\
\hline Exposure to violence & $\begin{array}{c}.18^{* * *} \\
(N=1,485)\end{array}$ & & $\begin{array}{c}.16^{* * *} \\
(N=1,930)\end{array}$ & $\begin{array}{c}.10^{* * *} \\
(N=1,924)\end{array}$ \\
\hline Aggressive fantasy & $\begin{array}{c}.16^{* * *} \\
(N=1,493)\end{array}$ & $\begin{array}{c}.27^{* * *} \\
(N=1,466)\end{array}$ & & $\begin{array}{c}.30^{* * *} \\
(N=2,517)\end{array}$ \\
\hline Normative beliefs aggression & $\begin{array}{c}.26^{* * *} \\
(N=1,495)\end{array}$ & $\begin{array}{c}.18^{* * *} \\
(N=1,467)\end{array}$ & $\begin{array}{c}.24^{* * *} \\
(N=1,508)\end{array}$ & \\
\hline
\end{tabular}

${ }^{*} p<.05 .{ }^{* *} p<.01 .{ }^{* * *} p<.001$.

As Table 4 shows, in both the early grades (e.g., Grade 2 because data on exposure to violence was limited in Grade 1) and the later grades (e.g., Grade 6), aggressive behavior, exposure to violence, normative beliefs, and aggressive fantasy all intercorrelated significantly though modestly. Generally, these correlations indicate that at any point the more aggressive child is one who is reporting more exposure to violence, more aggressive fantasizing, and normative beliefs that are more approving of aggression. The correlations of the social cognitions with exposure to violence are higher in the sixth grade: .18 and .27 compared with .10 and .16 in the early grades. Again, this is consistent with our developmental hypothesis that social cognitions crystallize in middle childhood. At the same time, the modest magnitude of the correlations suggests there is substantial individual variation on these measures that is not be related to exposure to violence. One possibility could be that some of the variance is due to the various interventions that about $75 \%$ of the sample was exposed to during this study. However, a comparison of these correlations in Table 4 within the $25 \%$ control (i.e., no intervention) subsample and within the rest of the sample showed no significant differences, making it unlikely that such interventions changed the relations much.

\section{Lagged Effects of Exposure to Violence on Social Cognitions and Aggression}

To examine the lagged effects of exposure to violence on aggression and social cognition as well as any reciprocal lagged effects of aggression and social cognitions on exposure to violence, we conducted an expanded series of HLM growth curve analysis with lagged effects added to the models.
Again, because of the pattern of missing observations shown in Table 1, HLM growth curve analysis is the optimal robust technique for analyzing the data from all cohorts and all grades simultaneously (Raudenbush \& Chan, 1992). Thus, we introduced time-lagged effects at Level 1, introduced gender as a child variable at Level 2 , and introduced school at Level 3 in these models to test for gender differences in the relations. For example, the growth model relating aggression to prior violence exposure was:

$$
\begin{gathered}
\operatorname{Agg}(t)=P 0+P 1^{*} \operatorname{Grade}(t)+P 2^{*} \operatorname{Agg}(t-1) \\
+P 3^{*} \operatorname{ViolExpos}(t-1)+\text { error } \\
P i=B i 0+B i 1^{*} \text { Gender }+ \text { error between children } \\
B i j=G i j 0+\text { error between schools. }
\end{gathered}
$$

The reciprocal model relating violence exposure to prior aggression was:

$$
\begin{array}{rl}
\operatorname{ViolExpos}(t)= & P 0+P 1^{*} \operatorname{Grade}(t) \\
& +P 2^{*} \operatorname{ViolExpos}(t-1)+P 3^{*} \operatorname{Agg}(t-1) \\
& + \text { error } \\
P i=B i 0+B i 1 & * \text { Gender }+ \text { error between children } \\
B i j=G i j 0+\text { error between schools. }
\end{array}
$$

The models relating violence exposure to normative beliefs and aggressive fantasy to violence exposure were parallel to this one. The resulting calculated growth curves for the three cases are shown in Figures 1 through 3 . The estimated slope parameters of these growth curves in Figures 1 through 3 represent the best estimates of the average effects over all 5 years of the predictor variables in 1 year on the criterion variables in the next year, that is, effect for a 1-year lag.

As with the general growth curves, on most of these lagged curves the intercepts varied with gender but the slopes did not. Males score higher on the intercept of 

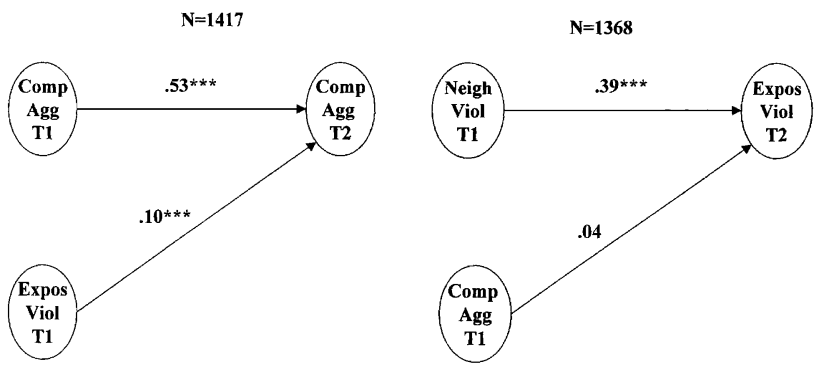

Figure 1. The average one-year lagged relation between exposure to neighborhood violence and aggressive behavior for Grades 1 to 6 computed from a three-level (time within person within school) hierarchical linear growth model. Comp Agg = composite aggression; $\operatorname{Exp}$ Viol = exposure to violence; Weigh Viol = neighborhood violence.

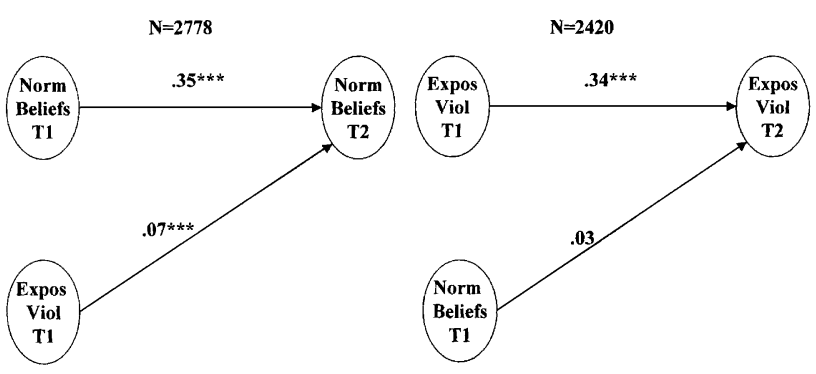

Figure 2. The average one-year lagged relation between exposure to neighborhood violence and normative beliefs approving of aggression for Grades 1 to 6 computed from a three-level (time within person within school) hierarchical linear growth model. Norm Beliefs $=$ normative beliefs; Expos Viol=exposure to violence.

the lagged growth curve relating violence exposure and prior normative beliefs to subsequent normative beliefs, $B 01=.31, t(2776)=3.16, p<.01$, and on the intercept relating violence exposure and prior aggressive fantasy to subsequent aggressive fantasy, $B 01=.41, t(2777)=3.80, p<.001$. Males did not score significantly higher on the intercept of the curve relating violence exposure and prior aggression to subsequent aggressive behavior, $B 01=.20, t(1415)=$ 1.54 , ns. These intercept effects simply indicate that males have initially higher scores on the social cognitions supporting aggression and continue to maintain those differences as they grow up. The fact that the intercept effect for gender on aggression was not significant is probably a function of the very high continuity of aggression over time within both genders. It means that gender does not add to the prediction of a participant's aggression in a particular grade if his or her aggression in the prior grade is known. Gender had a significant effect on slope of growth only for violence exposure predicting aggressive fantasy, $t(3655)=2.12, p<.05$. The effect of prior exposure to violence on fantasizing about aggression was greater

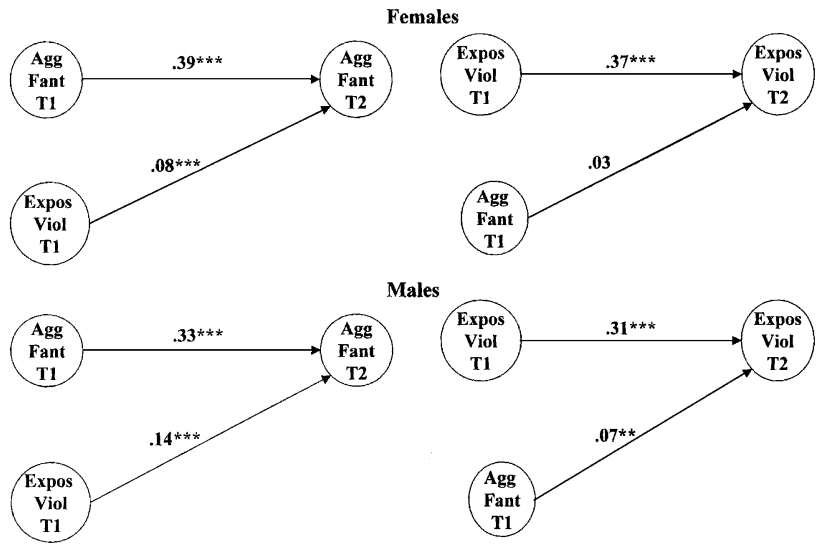

Figure 3. The average one-year lagged relation between exposure to neighborhood violence and aggressive fantasy for Grades 1 to 6 computed separately for males and females from a three-level (time within person within school) hierarchical linear growth model. Agg Fant=aggressive fantasy; Expos Viol= exposure to violence.

for males. Thus, we present the results for males and females separately only for aggressive fantasy.

Figures 1 through 3 reveal that across Grades 1 to 6 exposure to violence has a significant effect in increasing subsequent aggression, $B 30=.10$, $t(1503)=4.89, p<.001$; subsequent beliefs supporting aggression, $B 30=.07, t(18)=4.05, p<.001$; and aggressive fantasy, $B 30=.14, p<.001$ for males, $B 30=.08, p<.001$ for females. There is no effect of being aggressive on subsequent exposure to neighborhood violence or of approving of aggression on subsequent exposure to neighborhood violence. For males only, there is a significant effect of aggressive fantasizing on subsequent exposure to violence, $B 30=.07, p<.01$, but the magnitude of the effect is only half as great as the stimulating effect of exposure to violence on aggressive fantasizing. Overall, these results show that childhood exposure to violence predicts subsequent social cognitions about aggression and aggressive behavior for both males and females across Grades 1 to 6 .

In Figures 4 through 6, the lagged coefficients for these same calculated growth models are displayed separately for Grades 1 to 3 and Grades 4 to 6 . Males and females are analyzed together for aggressive fantasy as they showed the same effects for exposure to violence on fantasy. As we had hypothesized, exposure to violence predicts subsequent aggressive behavior both in the early grades, $B 30=.08$, $t(333)=2.22, p<.05$, and in the later grades, $B 30=.09, t(374)=2.18, p<.05$. However, the effects of exposure to violence on the social cognitions are only significant in the later grades: normative beliefs, $B 30=.13, t(407)=3.09, p<.01$, and aggressive fan- 


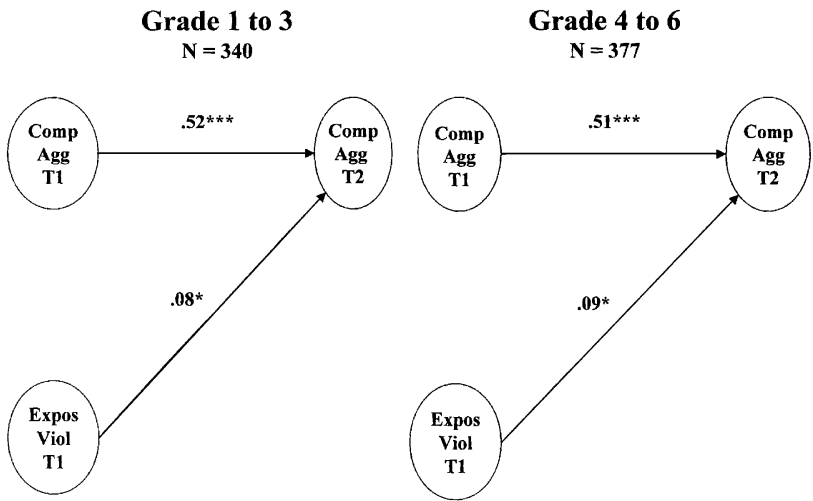

Figure 4. The average one-year lagged relations between exposure to neighborhood violence and aggressive behavior estimated separately for early and late elementary grades from a three-level hierarchical linear growth model. Comp Agg = composite aggression; Expos Viol = exposure to violence.
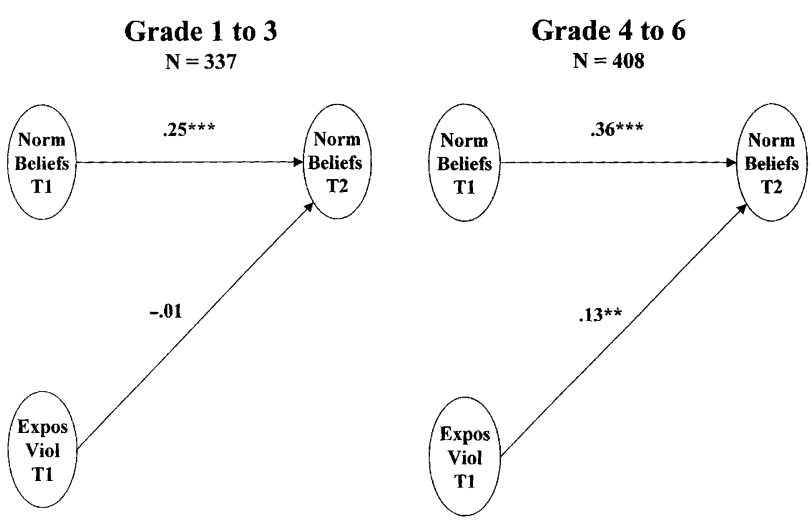

Figure 5. The average one-year lagged relations between exposure to neighborhood violence and normative beliefs approving of aggression estimated separately for early and late elementary grades from a three-level hierarchical linear growth model. Norm Beliefs $=$ normative beliefs; Expos Viol $=$ exposure to violence.

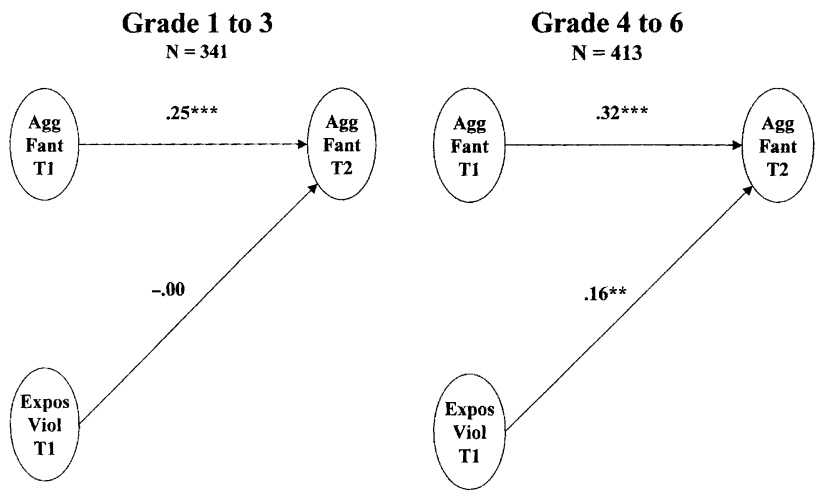

Figure 6. The average one-year lagged relations between exposure to neighborhood violence and aggressive fantasy estimated separately for early and late elementary grades from a three-level hierarchical linear growth model. Agg Fant=aggressive fantasy; Expos Viol $=$ exposure to violence. tasy, $B 30=.16, t(412)=3.49, p<.01$. Although the reciprocal models predicting exposure to violence could not be computed for the early grades because of the amount of missing data (see Table 1), they were computed for the older grades and showed no significant effects of aggression, normative beliefs supporting aggression, or aggressive fantasy on subsequent exposure to violence in this grade range. The conclusion these data suggest is that exposure to violence in the neighborhood is stimulating aggression in all grades and social cognitions supporting aggression in later middle childhood.

\section{Do Social Cognitions Mediate the Effects of Exposure to Violence on Aggression?}

Mediation can best be tested with structural models relating three waves of data (Kline, 1998); therefore, the causal ordering of the measured variables is unambiguous. For Grades 4 to 6 where prior exposure to violence was related to subsequent aggression and social cognitions, there were sufficient complete data to construct such models. In the early grades there were not sufficient data, but as shown earlier, exposure to violence did not predict subsequent aggressive fantasizing or normative beliefs in those grades. To maximize the sample size, we used the mean exposure to violence that the participants reported in the 24 months before their assessment on the social cognitions as the measure of exposure to violence. We used the composite teacher and peer-nominated measure of aggression taken 12 months after the assessment of the social cognitions as the criterion.

In left panel of Figure 7, the structural model is presented that tests the extent to which the effects of exposure to neighborhood violence on aggression are mediated by normative beliefs approving of aggression and by fantasizing about aggression. The parameters of this model were estimated using the entire sample, and they revealed that about $22 \%$ of the effect of exposure to violence on aggression was mediated by the two social cognitions. In particular, the mediating effect of normative beliefs, $16 \%$, $z=4.96, p<.001$, was highly significant. The mediating effect of aggressive fantasy, in contrast, was marginal, $6 \%, z=1.84, p<.10$. In the right panel of Figure 7 , the parameters for the structural model were recomputed for the control group subsample alone as a cross-validation. This cross-validation is important because the interventions to which the participants were exposed might have produced the mediation effect. However, the mediation effect was no smaller for the control group; in fact, it was 

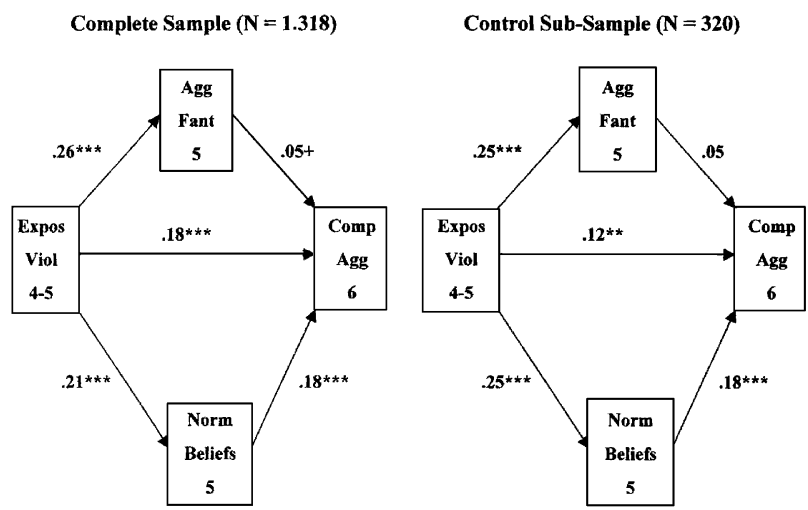

Figure 7. Two structural models showing the role of aggressive fantasy and normative beliefs in mediating the influence of exposure to community violence on aggressive behavior in Grades 4 to 6 . The model in the left panel fits the data from the entire sample. For this model the total effect of exposure to violence on aggression $=.234$; the mediated effect through aggressive fantasy $=.013=6 \%, p<.10$; the mediated effect through normative beliefs $=.037=16 \%, p<.001$; and the leftover direct effect of exposure to violence $=.184=78 \%, p<.001$. The model in the right panel fits the data from the control group subsample. For this model the total effect of exposure to violence on aggression $=1.70$ the mediated effect through aggressive fantasy $=.012=7 \%$, $p<.10$; the mediated effect through normative beliefs $=$ $.043=25 \%, p<.001$; and the leftover direct effect of exposure to violence $=.115=68 \%, p<.001$. Agg Fant $=$ aggressive fantasy; Comp Agg = composite aggression; Norm Beliefs = normative beliefs; Expos Viol = exposure to violence.

slightly larger, with about $32 \%$ of the effect of exposure to violence on aggression being mediated by social cognitions. The fact that mediation is no less in the control group subsample suggests that mediation is not a consequence of the interventions many participants experienced. Again, normative beliefs was the social cognition that accounted for most of the mediating effect, $25 \%, z=2.53, p<.01$.

Although these models suggest that the majority of the effect of exposure to neighborhood violence on aggression is due to processes not related to the two cognitions measured in this study, the models confirm that normative beliefs play an important mediating role connecting exposure to contextual violence to subsequent aggressive behavior in older elementary school children.

\section{Discussion}

In the present study we examined the effects of community violence exposure on the aggressive cognitions and behavior of elementary-school-age children living in urban and inner-city communities. The first question we addressed was whether exposure to community violence, aggressive cognitions, and aggressive behavior increase or decrease on average during the elementary school years. We found that children reported a decrease in exposure to community violence over time that was not explained by changing neighborhood environments. Several explanations seem plausible. First, given the generally high rates of community violence in the neighborhoods where children in our study live, repeated exposure may result in habituation to the point that children are less likely to notice and pay attention to incidents of violence. Along these lines, some of this decline might reflect measurement error, with younger children more likely to exaggerate their experiences than older children. It may also be that as children get older they are also less likely to play outside; therefore, they are simply less likely to be in settings where neighborhood violence occurs. It is plausible that this would be particularly true for females who indeed showed a greater decline in exposure.

In contrast to our findings for violence exposure, aggressive cognitions and aggressive behavior were found to increase during the elementary school years. This is consistent with studies showing increases in aggression with age during childhood and adolescence (Elliott, 1994; Loeber \& StouthamerLoeber, 1998). Although Tremblay (2000) pointed out that different types of aggression may follow different developmental courses in different settings, our study showed that during the early elementary school years in urban and inner-city settings, children are developing patterns of behavior that are increasingly likely to involve aggressive interchanges. These patterns of behavior are also accompanied by changes in cognitions related to aggressive scripts that provide opportunities to rehearse cognitively aggressive sequences and demonstrate greater support for its legitimacy. Otherwise put, as children get older they are acting more aggressively and are developing cognitions that support such behavior.

Our theoretical model emphasized the importance of internal self-regulating processes that become more stable over time. Thus, we expected that characteristic patterns of thought and action would be more pronounced as children got older. This was the case with aggressive behavior. Consistent with previous research, aggressive behavior showed high continuity across all grades and across multiple years. Aggressive fantasy and normative beliefs about aggression also showed reasonably high year-to-year continuity that increased with age. However, cognitions seemed to be much less stable than aggressive behavior over multiple years. This points to the importance of focusing preventive 
interventions during the elementary school years on influencing children's emerging cognitive structures.

Our data also provide additional support for the well-documented relation between social cognition and aggression. More aggressive children report more exposure to violence, aggressive fantasizing, and normative beliefs supporting aggression. In the present study, we were particularly interested in the effects of exposure to community violence on the development of these cognitive and behavioral patterns. Although previous studies have demonstrated a link between harsh or violent family environments and aggressive social cognition (e.g., Weiss et al., 1992), few studies have examined the effect of community violence exposure on these cognitive factors and whether these effects vary by age.

Perhaps the most important finding from this study is that exposure to community violence increases children's subsequent aggressive behavior and social cognition supporting aggression. Violence exposure seemed to stimulate subsequent aggressive behavior for children in both the early and late elementary grades. However, violence exposure only stimulated increases in aggressive social cognition during the later elementary school grades. In previous research with urban and inner-city youth, we found that a child's own aggressive behavior predicted aggressive cognitions during the early elementary school years, with cognitions predicting behavior during the later elementary school years. We interpreted these findings as showing that children's cognitions emerged from their observations of their own behavior early on, with cognitions then guiding behavior as patterns of thought become more stable during the later elementary school years. Based on these findings, we had expected that observation of community violence would predict increases in aggressive cognitions during the early elementary years as well. This does not seem to have been the case. It may be that the effects of community violence exposure on cognition are related to a habituation process that requires more time than behavioral changes require. As social cognitions are unstable during the early elementary years, the effects of exposure on cognition are thus less noticeable.

Although exposure to violence predicted subsequent aggression and social cognition supporting aggression, the reverse was generally not true: Exposure was not predicted by prior aggression or social cognition. One exception was a small but significant effect for males of aggressive fantasizing on subsequent exposure to violence. This could indicate that males who fantasize more about aggressive interactions seek out opportunities to observe them or at least do not avoid them to the extent that less aggressive males avoid them. This process might begin during childhood as children observe violence, behave aggressively, come to see it as a legitimate behavior, prepare for future aggression via cognitive fantasy rehearsal, and seek out opportunities to view and observe evidence of their emerging world view that involves aggressive interchanges. Furthermore, because they have fantasized about it so much, they may be less afraid of exposing themselves to violent situations. By adolescence, children who are thus primed for aggression may gravitate toward other contexts such as gangs that provide ready access to observation of and participation in violence.

Given the observed relations among exposure to community violence, social cognition, and aggression during the later elementary years, we also examined whether the effects of violence exposure were mediated by social cognition. We found that normative beliefs did play a significant mediating role. As discussed by other researchers (e.g., Schwab-Stone et al., 1995), viewing violence as normative may desensitize children to its true consequences and create a context whereby it is accepted as a way of life, resulting in an increased readiness to behave aggressively. Still, much of the effect of exposure to violence was not mediated in this way. That result is not surprising, as numerous other potential mediators that were unmeasured could play a role.

Consistent with the literature on gender differences in physical aggression, males scored higher than females on measures of aggression, fantasizing about aggression, and normative beliefs approving of aggression. Although the growth in aggression and normative beliefs approving of aggression during the elementary school years did not differ between boys and girls, girls did display more of an increase than boys in aggressive fantasy. This may reflect differential socialization by gender in terms of the appropriateness of aggression; that is, for girls, aggression may be relegated to the domain of fantasy and other private thought processes. Differential socialization may also explain why girls showed more exposure to community violence initially and more of a decrease in exposure to community violence over time. Females may be socialized to notice violence more, but may also be socialized to avoid violent contexts more as they grow up.

For the major relations of interest in this study, however, gender played no significant moderating 
role. The correlations between exposure to community violence, aggressive behavior, and aggressive cognitions did not vary consistently by gender. The effects of exposure to violence on subsequent aggression and aggressive cognitions were not significantly different for boys and girls, although relations between violence exposure and aggressive fantasy were stronger for boys than girls. It may be that both boys and girls retreat to aggressive fantasies as a mechanism for coping with high levels of witnessing violence, but boys may take this a step further because they have a greater expectation that they will be involved in or around violence. Finally, normative beliefs mediated the relation between exposure to violence and aggressive behavior in the later elementary grades for both boys and girls.

Our study was limited to the effects of witnessing neighborhood violence on children's aggressive behavior and cognition. However, even in the high-violence communities where children in this study lived, this type of violence (e.g., shootings, beatings) is still relatively rare. Furthermore, our assessment was limited to a few self-report items that referred to this extreme type of violence. Although the level of violence exposure reported by children seemed to concur with official crime data, this is still a relatively weak measure that taps exposure only to extreme events.

It is also important to examine the extent to which other types of violence exposure that are likely to be more regularly experienced are also influencing children's development and how the effect of witnessing violence varies as a function of type and level of exposure. This includes family violence, violence in the peer group, and violence in the classroom and school. It may be that the effects of violence exposure are additive, so that witnessing more violence across contexts has a cumulative effect on behavior and cognition. Alternately, the effects of violence exposure may be multiplicative, so that the compound effects are particularly detrimental.

Future longitudinal research should focus on the influence of multiple types of violence exposure on children's behavior and the potential mediating mechanisms. One suggested line of research is to expand the potential social cognitive mediators studied to include a broader range of factors. For instance, it may be that violence exposure also influences outcome expectancies, with increased exposure to the real consequences of violence (e.g., pain, injury) leading to more negative outcome expectancies. On the other hand, repeated violence exposure might lead children to adopt revenge goals to ward off potential threats. These relations may also be moderated by age and gender, as we found in the current study. Other suggested moderators might include children's level of emotionality and level of aggression. For instance, violence exposure might have the greatest effect for children who are marginally aggressive and may be more susceptible to social influence (Dodge, Nicholson, \& Stearns, 2003).

Beyond a focus on social cognitive mediators of violence exposure, it is important to examine and compare the effects of both exposure and victimization. It seems that both experiences negatively affect children; however, it is important to differentiate further the unique contributions of exposure versus victimization, as well as the joint influence of being both victimized and exposed to violence. Just as it is important to examine the influence of exposure to different types of violence, it is also important to compare the influence of different types of violent victimization (e.g., by peers, by family members, by strangers).

It goes with out saying that the first line of prevention is to reduce the levels of violence in the community and elsewhere to minimize children's exposure to violence and to increase their safety. However, to the extent that children continue to observe violence, it is also critical to recognize that it does have a significant and harmful impact on their development and contributes to the learning of aggression. How can we minimize the impact of violence exposure on behavior and cognition? As mentioned previously, the results of our own intervention study in urban communities and of other similar studies suggest that efforts to modify and redirect children's cognitions can be successful if begun early. These efforts should be designed to lessen habituation to violence, so that children continue to see it as harmful and non-normative. These efforts should also focus on teaching children nonaggressive scripts that are sensitive to the difficult and often violent environments that they must navigate, as well as engaging parents and others in protecting children from the potentially harmful effects of violence exposure.

\section{References}

Achenbach, T. M. (1978). The child behavior profile: I. Boys aged 6-11. Journal of Consulting and Clinical Psychology, $46,478-488$.

Achenbach, T. M. (1991). Integrative guide for the 1991 CBCL/ 4-18, YSR, and TRF profiles. Burlington: University of Vermont, Department of Psychiatry.

Attar, B. K., Guerra, N. G., \& Tolan, P. H. (1994). Neighborhood disadvantage, stressful life events, and 
adjustment in urban elementary-school children. Journal of Clinical Child Psychology, 23, 391-400.

Bandura, A. (1973). Aggression: A social learning analysis. New York: Holt.

Bandura, A. (1986). Social foundations of thought and action: A social-cognitive theory. Englewood Cliffs, NJ: Prentice Hall.

Bell, C. C., \& Jenkins, E. J. (1991). Traumatic stress and children. Journal of Health Care for the Poor and Underserved, 2, 175-185.

Bryk, A. S., \& Raudenbush, S. W. (1987). Application of hierarchical linear models to assessing change. Psychological Bulletin, 101, 147-158.

Dempsey, M. (2002). Negative coping as mediator in the relation between violence and outcomes: Inner city African American youth. American Journal of Orthopsychiatry, 72, 102-109.

Dodge, K. A., Bates, J. E., \& Pettit, G. A. (1990). Mechanisms in the cycle of violence. Science, 250, $1678-1683$.

Dodge, K. A., Nicholson, M. J., \& Stearns, M. E. (2003). From mischief to violence: How culture shapes the growth of aggressive behavior. Paper presented at the biannual meeting of the Society for Research in Child Development, Tampa, FL.

Eisenberg, N., Fabes, R. A., Nyman, M., Bernzweig, J., \& Pinuelas, A. (1994). The relations of emotionality and regulation to children's anger-related reactions. Child Development, 65, 109-128.

Elliott, D. S. (1994). Serious violent offenders: Onset, developmental course and termination: The American Society of Criminology 1993 Presidential Address. Criminology, 32, 1-21.

Eron, L. D. (1987). The development of aggressive behavior from the perspective of a developing behaviorism. American Psychologist, 42, 435-442.

Eron, L. D., Walder, L. O., \& Lefkowitz, M. M. (1972). The learning of aggression in children. Boston: Little, Brown.

Flannery, D. J., Singer, M. I., \& Wester, K. (2001). Violence exposure, psychological trauma, and suicide risk in a community sample of dangerously violent adolescents. Journal of the American Academy of Child and Adolescent Psychiatry, 40, 1-8.

Garbarino, J., Kostelny, K., \& Dubrow, N. (1991). What children can tell us about living in danger. American Psychologist, 46, 376-382.

Guerra, N. G., Huesmann, L. R., Tolan, P. H., Van Acker, R., \& Eron, L. D. (1995). Stressful events and individual beliefs as correlates of economic disadvantage and aggression among urban children. Journal of Consulting and Clinical Psychology, 63, 518-528.

Huesmann, L. R. (1988). An information processing model for the development of aggression. Aggressive Behavior, $14,13-24$.

Huesmann, L. R. (1998). The role of social information processing and cognitive schema in the acquisition and maintenance of habitual aggressive behavior. In R. E. Geen \& E. Donnerstein (Eds.), Human aggression:
Theories, research, and implications for policy (pp. 73109). New York: Academic Press.

Huesmann, L. R., Eron, L. D., Lefkowitz, M. M., \& Walder, L. O. (1984). Stability of aggression over time and generations. Developmental Psychology, 20, 1120-1134.

Huesmann, L. R., \& Guerra, N. G. (1997). Children's normative beliefs about aggression and aggressive behavior. Journal of Personality and Social Psychology, 72, $408-419$.

Huesmann, L. R., Guerra, N. G., Eron, L. D., \& Crawshaw, V. B. (1994). Measuring children's aggression with teachers' predictions of peer nominations. Psychological Assessment, 6, 329-336.

Huesmann, L. R., Guerra, N. G., Miller, L., \& Zelli, A. (1992). The role of social norms in the development of aggression. In H. Zumckly \& A. Fraczek (Eds.), Socialization and aggression (pp. 139-151). New York: Springer-Verlag.

Kline, R. (1998). Structural modeling. New York: Guilford.

Loeber, R., \& Stouthamer-Loeber, M. (1998). Development of juvenile aggression and violence. Some common misconceptions and controversies. American Psychologist, 53, 242-259.

Lynch, M., \& Cicchetti, D. (1998). An ecological-transactional analysis of children and contexts: The longitudinal interplay among child maltreatment, community violence, and children's symptomatology. Development and Psychopathology, 10, 235-257.

Marans, S., \& Cohen, D. (1993). Children and inner-city violence: Strategies for intervention. In L. Leavitt \& N. Fox (Eds.), Psychological effects of war and violence on children (pp. 281-302). Hillsdale, NJ: Erlbaum.

Martinez, P., \& Richters, J. E. (1993). The NIMH Community Violence Project: II. Children's distress symptoms associated with violence exposure. Psychiatry: Interpersonal and Biological Processes, 56, 22-35.

Metropolitan Area Child Research Group. (2002). A cognitive-ecological approach to preventing aggression in urban settings: Initial outcomes for high-risk children. Journal of Consulting and Clinical Psychology, 70, 179-194.

Ng-Mak, D. S., Salzinger, S., Feldman, R., \& Stueve, A. (2002). Normalization of violence among inner-city youth: A formulation for research. American Journal of Orthopsychiatry, 72, 92-101.

Olweus, D. (1979). Stability of aggressive reaction patters in males: A review. Psychological Bulletin, 85, 852-875.

Osofsky, J. D. (1995). The effects of exposure to violence on young children. American Psychologist, 50, 782-788.

Osofsky, J. D., Wewers, S., Hann, D. M., \& Fick, A. C. (1993). Chronic community violence: What is happening to our children? Psychiatry, 56, 36-45.

Pynoos, R. S. (1993). Traumatic stress and developmental psychopathology in children and adolescents. In J. M. Oldham, M. B. Riba, \& A. Tasman (Eds.), American Psychiatric Press review of psychiatry, Vol. 12. (pp. 205238). Washington, DC: American Psychiatric Press. 
Raudenbush, S. W., \& Chan W-S. (1992). Growth curve analysis in accelerated longitudinal designs. Journal of Research in Crime and Delinquency, 29, 387-411.

Richters, J. E., \& Martinez, P. (1993). The NIMH community violence project: Vol. 1. Children as victims of and witnesses to violence. Psychiatry, 56, 7-21.

Rivera, B., \& Widom, C. S. (1990). Childhood victimization and violent offending. Violence and Victims, 5, 19-35.

Rogosa, D., Brandt, D., \& Zimowski, M. (1982). A growth curve approach to the measurement of change. Psychological Bulletin, 92, 726-748.

Rosenfeld, E., Huesmann, L. R., Eron, L. D., \& TorneyPurta, J. V. (1982). Measuring patterns of fantasy behavior in children. Journal of Personality and Social Psychology, 42, 347-366.

Schwab-Stone, M., Ayers, M. E., Kasprow, W., Voyce, C., Barone, C., \& Shriver, T., et al. (1995). No safe haven: A study of violence exposure in urban communities. Journal of the American Academy of Child and Adolescent Psychiatry, 34, 1343-1352.

Schwab-Stone, M., Chen, C., Greenberger, E., Silver, D., Lichtman, J., \& Voyce, C. (1999). No safe haven II: The effects of violence exposure on urban youth. Journal of the American Academy of Child and Adolescent Psychiatry, 38, 359-367.

Schwartz, D., \& Proctor, L. J. (2000). Community violence exposure and children's social adjustment in the school peer group: The mediating roles of emotional regulation and social cognition. Journal of Consulting and Clinical Psychology, 68, 670-683.

Singer, M. I., Anglin, T. M., Song, . L. Y., \& Longhofer, L. (1995). Adolescents' exposure to violence and associated symptoms of psychological trauma. JAMA, 273, $477-482$.

Singer, M. I., Miller, D. B., Guo, S., Flannery, D. J., Frierson, T., \& Slovak, K. (1999). Contributors to violent behavior among elementary and middle school children. Pediatrics, 104, 878-884.

Spacarelli, S., Coatsworth, J. D., \& Bowden, B. S. (1995). Exposure to serious family violence among incarcerated boys: Its association with violent offending and potential mediating variables. Violence and Victims, 10, $163-182$.

Tremblay, R. (2000). The development of aggressive behaviour during childhood: What have we learned in the past century? International Journal of Behavior Development, 24, 129-141.

Weiss, B., Dodge, K. A., Bates, J. E., \& Pettit, G. A. (1992). Some consequences of early harsh discipline: Child aggression and a maladaptive social-information-processing style. Child Development, 63, 1321-1335.

Widom, K. (1989). Does violence beget violence? Psychological Bulletin, 106, 3-28. 